\title{
PROJETO DE EXTENSÃO: HUMANIZAÇÃO E EDUCAÇÃO EM SAÚDE JUNTO AOS CUIDADORES, CRIANÇAS E ADOLESCENTES NO HOSPITAL UNIVERSITÁRIO INFANTIL
}

\author{
Bruna Cavalheiro Engel ${ }^{1}$ \\ Caroline da Silva ${ }^{2}$ \\ Claiza Barretta ${ }^{3}$ \\ Francielly Alexandre Fabris ${ }^{4}$ \\ Luana Bertamoni Wachholz ${ }^{5}$ \\ Marcia Aparecida Miranda de Oliveira ${ }^{6}$ \\ Maria Eduarda Luz $^{7}$ \\ Mariana Francisco da Silva ${ }^{8}$ \\ Thalia Nathalia dos Santos ${ }^{9}$ \\ Willian Bueno de Oliveira ${ }^{10}$ \\ Fabíola Hermes Chesani ${ }^{11}$
}

Resumo: O presente artigo é um relato de experiência do projeto de extensão Humanizar e Educar em saúde. O projeto é constituído por uma equipe interdisciplinar, contando com docentes e discentes dos cursos de Fisioterapia, Psicologia e Nutrição. O projeto de extensão é voltado à humanização e educação em saúde no Hospital Universitário Pequeno Anjo (HUPA), que atende crianças de 0 a 14 anos. Este projeto tem como objetivo a troca de saberes junto às crianças, adolescentes e cuidadores por meio de roda de conversa e estratégias dinâmicas. A metodologia adotada é a da roda de conversa orientada pelos preceitos de Paulo Freire. No período de março de 2015 a junho de 2016 aconteceram 90 encontros e a participação de 742 cuidadores, crianças e adolescentes. As rodas de conversa com cuidadores de crianças e adolescentes permitiu a troca de experiências, na qual os sujeitos auxiliam uns aos outros no processo de enfrentamento da hospitalização.

Palavras-chave: Humanização; Educação em saúde; Projeto de extensão; Saúde coletiva.

\footnotetext{
${ }^{1}$ Psicologia/Universidade do Vale do Itajaí, Brasil. brunaengel@edu.univali.br.

2 Fisioterapia/Universidade do Vale do Itajaí, Brasil. caroline.silva3006@gmail.com.

${ }^{3}$ Nutrição/Universidade do Vale do Itajaí. Brasil. claizabarretta@yahoo.com.br.

${ }^{4}$ Nutrição/Universidade do Vale do Itajaí. Brasil. franfabris2010@hotmail.com.

${ }^{5}$ Nutrição/Universidade do Vale do Itajaí. Brasil. Iuana.bertamoni@hotmail.com.

${ }^{6}$ Psicologia/Universidade do Vale do Itajaí, Brasil. marciaoliveira3@terra.com.br.

${ }^{7}$ Nutrição/Universidade do Vale do Itajaí. Brasil. mariaeduardaluzz@gmail.com.

${ }^{8}$ Psicologia/Universidade do Vale do Itajaí, Brasil. psicomarianafs@gmail.com.

${ }^{9}$ Fisioterapia/Universidade do Vale do Itajaí, Brasil. thalia-bc@hotmail.com.

${ }^{10}$ Psicologia/Universidade do Vale do Itajaí, Brasil. bueno.will2@gmail.com.

${ }^{11}$ Fisioterapia/Universidade do Vale do Itajaí, Brasil. fabiola.chesani@univali.br.
} 